\title{
Rastreamento do câncer de mama: as três luzes do semáforo
}

\author{
Screening for breast cancer: the three traffic lights
}

Rastreo del cáncer de mama: las tres luces del semáforo

José Agostinho Santos. Unidade de Saúde Familiar Lagoa, Senhora da Hora, Portugal. zeagostinho@hotmail.com (Autor correspondente)

\section{Resumo}

A interpretação do rastreamento do câncer de mama como um semáforo com três luzes convida a uma revisão rápida da evidência científica e à retenção dos principais pontos que esse procedimento revela. As recomendações a favor do rastreamento são produzidas por autores que conferem a ele uma luz verde, de avanço, porque acreditam que o saldo risco/benefício é favorável a sua aplicação em mulheres em idades-alvo e com risco médio. Mas, tal como a própria expressão risco/benefício sugere, existe um risco que deverá ser levado em conta no início do rastreamento, um alerta que assume a luz vermelha. É notório, pela evidência avaliada por autores independentes, que existe uma relação entre prós e contras (luz amarela). 0 papel de salvaguardar o equilíbrio dessa balança pertencerá, talvez, ao médico de família, cabendo, então, em última instância, à mulher em idadealvo colocar o último peso sobre um dos dois pratos.

\section{Abstract}

The interpretation of breast cancer screening as three different traffic lights invites the reader for a quick review of the scientific evidence and the retention of the main points that this procedure reveals. The recommendations for the screening are produced by authors that give a green light for the screening advance, because they believe that the risk/benefit balance is favorable to its application in women at the target ages and average risk. However, as the expression "risk/benefit" implies, there is a risk that must be taken into account at the time of starting the screening, which assumes an alert for the red light. It is clear, from the evidence evaluated by independent authors, that there is a balance of pros and cons (yellow light). The role of ensuring the balance of this scale belongs perhaps to the General Practitioner and the role of putting the last weight on one of the two plates belongs to the women at the target-ages to screening.

\section{Resumen}

La interpretación del rastreo de cáncer de mama como un semáforo con tres luces invita a una rápida revisión de la evidencia científica y a la retención de los principales puntos que este procedimiento revela. Las recomendaciones para el rastreo son producidas por autores que dan luz verde para avanzar, porque creen que el balance riesgo/beneficio es favorable a su aplicación en mujeres en edad de riesgo pero con riesgo medio. Pero, como la misma expresión "riesgo/beneficio" implica, existe un riesgo que se debe tener en cuenta al inicio del rastreo, un alerta que asume la luz roja. Es claro, a partir de la evidencia evaluada por autores independientes, que existe una relación entre pros y contras (luz amarilla). El rol de salvaguardar el equilibrio de esta balanza pertenecerá, quizás, al Médico de Familia, cabiendo, entonces en última instancia, a la mujer en edad de riesgo el papel de poner el último peso sobre uno de los dos platos.

\section{Palavras-chave:}

Neoplasias da Mama Programas de Rastreamento Análise Custo-benefício Prevenção Quaternária
Keywords:

Breast Neoplasms Mass Screening Cost-benefit Analysis Quaternary Prevention

Palabras clave: Neoplasias de la Mama Programa de Rastreo Análisis Costo-beneficio Prevención Cuaternaria 


\section{Rastreamento do câncer de mama: 0 saldo risco/benefício}

Nos últimos anos, os rastreamentos oncológicos têm merecido destaque na literatura. Tal destaque é justificado pelo crescente número de publicações polêmicas que questionam a sua suposta boa relação custo/benefício, mesmo após décadas do início da sua implementação ${ }^{1-3}$. As conclusões desses estudos motivaram críticas dirigidas à metodologia e limitaçóes dos ensaios que, por sua vez, levaram à edição de artigos de opinião em resposta aos comentários primários ${ }^{4-6}$.

Tendo como ponto de partida uma época em que proliferavam tumores avançados e letais, frequentemente inoperáveis mediante as técnicas da época, alguns rastreamentos oncológicos (como o do câncer de mama) seguiram o seu caminho até os dias atuais, com a detecção de uma série de tumores bem menores e operáveis. Tal multiplicação de intervençôes cirúrgicas gerou médicos experientes e aperfeiçoou técnicas que foram progressivamente mais bem-sucedidas na orientação terapêutica ${ }^{7,8}$. Assim nasceu uma onda de retroalimentação do entusiasmo relativo aos métodos de rastreamento, gradualmente mais sofisticados e efetivos na detecção precoce ${ }^{7}$. Foram elaboradas recomendaçôes das grandes sociedades científicas a favor de alguns rastreamentos e foram adotadas medidas governamentais que favorecem a adesão populacional ao torná-los substancialmente menos dispendiosos ou gratuitos para o cidadão comum ${ }^{9-11}$. Porém, no caso particular do rastreamento do câncer de mama por meio da mamografia, estudos bem realizados têm lançado resultados que resfriam os ânimos entre a comunidade médica. Por outro lado, embora possam não colocar em dúvida a efetividade da detecçáo precoce de tumores mamários e a redução da taxa de mortalidade específica, esses ensaios alertam para os custos que lhe são associados e que estão muito além dos custos financeiros: os custos humanos provenientes do sobrediagnóstico e do sobretratamento ${ }^{1,4,7}$.

Este breve artigo de perspectiva pretende apenas relembrar o Médico de Família e Comunidade (MFC) que, mesmo que disponha das mais diversas diretrizes recomendando o rastreamento sistemático do câncer da mama através de mamografia nas mulheres em idades-alvo, necessitará conhecer o lado potencialmente danoso que existe na atitude clínica proativa de encaminhamento da sua paciente para esse procedimento. Tal será tão simples como relembrar ou conhecer os principais efeitos colaterais de um fármaco prescrito que podem afetar a qualidade de vida da pessoa a quem presta cuidados.

A interpretação do rastreamento do câncer de mama como um semáforo com três luzes convida a uma revisão rápida da evidência científica. As recomendaçóes a favor do rastreamento são produzidas por um conjunto de autores que lhe confere uma luz verde para avanço porque, após análise da evidência, acreditam que o saldo risco/benefício é favorável a sua aplicação nas mulheres em idades-alvo e com risco médio [isto é, mulheres sem mutação do gene Breast Cancer (BRCA) conhecida, sem história familiar de câncer mamário ou ovariano associado a mutação BRCA+, sem história de irradiação torácica] provindas da população geral ${ }^{12}$. Mas, tal como a própria expressáo risco/benefício sugere, existe um risco que deverá ser levado em conta no início do rastreamento, um alerta que assume a luz vermelha. E relativamente ao screening de câncer de mama, diversas interpretaçóes da evidência estão patentes na literatura, interessando, para a Medicina de Família e Comunidade, as leituras de sociedades científicas de grande impacto e independentes, como a Cochrane Collaboration ou a US Preventive Services Task Force (USPSTF) ${ }^{13,14}$.

\section{Rastreamento do câncer de mama: a luz verde}

A revisão de 2009 da Cochrane Collaboration congregou, com rigor, os resultados de sete ensaios clínicos randomizados e controlados (envolvendo no total cerca de 600 mil mulheres) que compararam a taxa de mortalidade de um grupo de mulheres com idade entre 39 e 74 anos submetidas a mamografia de rastreamento com a de um grupo de mulheres não-submetidas ao exame, após 13 anos. Os autores consideraram que três desses ensaios possuíam boa qualidade metodológica (ensaios elaborados no Canadá, no Reino Unido e em Malmö, Suécia), enquanto que classificaram quatro ensaios com randomização subótima (ensaios de Göteborg, New York, Stockholm e Two-Country). Curiosamente, a meta-análise envolvendo os três primeiros não revelou uma redução significativa da taxa de mortalidade específica por carcinoma mamário [Risco Relativo $(\mathrm{RR})=0,90(0,79-1,02)]$, porém os restantes documentaram, em conjunto, um decréscimo com significância $[\mathrm{RR}=0,75$ $(0,67-0,82)]$. Os autores da Cochrane estimam um RR final de $0,85^{13}$.

Este RR não se situa muito longe do calculado pela USPSTF que, analisando os mesmos sete ensaios clínicos, aponta para um RR de 0,85 (0,75-0,96) para mulheres com idade entre 39 e 49 anos, 0,86 $(0,75-0,99)$ para as mulheres com idade entre 50 e 59 anos e de $0,68(0,54-0,87)$ para aquelas na faixa etária entre 60 e 69 anos. A USPSTF recomenda o rastreamento 
do câncer de mama através de mamografia bianual com força B (recomendação baseada em evidência inconsistente, de qualidade limitada e orientada para o paciente) para mulheres com idades entre 50 e $69 \operatorname{anos}^{14}$.

Assim, o benefício do screening é evidenciado nas publicaçôes desses grupos independentes, salientando uma reduçáo de cerca de 20\% na mortalidade por carcinoma mamário, embora sem tradução a nível da mortalidade por todas as causas. Os autores da revisão da Cochrane e do The Nordic Cochrane Centre transformaram este valor relativo em números absolutos e mais claros, destacando que 2 mil mulheres terão que ser rastreadas durante 10 anos para que uma morte por câncer de mama seja evitada ${ }^{7,13}$.

\section{Rastreamento do câncer de mama: a luz vermelha}

Entretanto, desde que os ensaios em foco tiveram início, o diagnóstico clínico e tratamento para o câncer mamário obtiveram grandes melhoramentos (quer em termos de recursos técnicos, quer sob o prisma dos recursos humanos). Para os membros do The Nordic Cochrane Centre, esse incremento qualitativo nesses dois níveis poderá atenuar o benefício do rastreamento acima referido, pois é especulável que mesmo as mulheres não submetidas a mamografia de rastreamento sejam diagnosticadas e sofram intervenções com sucesso após recorrerem a consulta médica por notarem alteraçáo nos seios (fruto de um novo conceito denominado breast awareness, cujas melhores traduçôes para português seriam autocuidado mamário ou autoconscientização mamária, que apela a um autoconhecimento corporal e a uma atenção particular da mulher a alteraçốes encontradas nas mamas durante a sua higiene íntima ou inspeção mamária) $)^{7,15}$.

Esse aspecto poderá ter um impacto ainda maior se tiver como pano de fundo o sobrediagnóstico e o sobretratamento, que retêm a maior carga dos riscos inerentes ao rastreamento através da mamografia. Esse exame radiográfico conduz a uma maior taxa de deteç̧ão de cânceres que cresceriam de forma lenta e que nunca se tornariam sintomáticos durante a vida da mulher (sobretudo, os carcinomas in situ). A esse fenômeno de identificação dessa fração da patologia se denomina sobrediagnóstico ${ }^{15,16}$. A percentagem desses tumores entre todos os cânceres detectados por esse método de rastreamento é estimada entre 19\% - e 30\%. Assim, os mesmos autores do The Nordic Cochrane Centre estimam que em 2 mil mulheres rastreadas regularmente durante 10 anos, 10 mulheres saudáveis terão o diagnóstico de um câncer mamário que nunca comprometeria a sua qualidade de vida. Esse diagnóstico conduziria a uma intervençâo cirúrgica nas mamas, na maioria dos casos, e, possivelmente, a radioterapia ou quimioterapia ${ }^{7}$.

Uma revisão mais recente elaborada pelo Independent UK Panel on Breast Cancer Screening aponta um diferencial menor para a realidade britânica (onde existe um mass screening) e atribui menor dimensão ao sobrediagnóstico. Calcula que, entre 10 mil mulheres britânicas convidadas para o rastreamento, serão realizados 681 diagnósticos de câncer de mama, dos quais 129 serão sobrediagnósticos e 43 serão cânceres fatais prevenidos pela detecção precoce ${ }^{1}$. As discrepâncias de cálculo devem-se à inclusão, nessa revisão, de estudos que foram rejeitados pelos autores da Cochrane e do USPSTF, alegando que a metodologia teria maiores limitaçôes ${ }^{13,14}$. Contudo, mesmo assim nessa revisão britânica o número de mulheres com sobrediagnóstico é três vezes superior às mulheres cuja morte por câncer de mama é prevenida. Sobressai ainda um grande conjunto de cerca de 500 mulheres com cânceres detetados pelo screening que se tornariam sintomáticos mas não fatais para as quais o benefício do rastreamento permanece sob dúvida.

Outra face dos riscos do rastreamento são os falso-positivos: em 2 mil mulheres rastreadas durante 10 anos, 200 terão falso-positivos potencialmente geradores de um dano psicológico imediato por receio de câncer ${ }^{7,13,14}$. Uma boa parte dessas mulheres será submetida a exames complementares de esclarecimento (incluindo biópsia) que agravam ainda mais o dano psicológico e acrescentam dano orgânico ${ }^{7,13}$. 


\section{Rastreamento do câncer de mama: a luz amarela}

De uma reflexão breve sobre os fatos acima apresentados surgem os dois lados desse procedimento preventivo: existe um benefício documentado, assim como emerge um dano que poderá surpreender o Médico de Família e para o qual ele precisará estar alerta.

De fato, a fomentação desse rastreamento por entidades governamentais por meio de patrocínios pelo estado ou por normas emitidas por delegaçôes de saúde estatais, ${ }^{9} 10,11,16$ é, por sua vez, promotora de uma sensação de segurança no médico prescritor do exame mamográfico, acreditando que o procedimento tem um grande benefício comprovado e um dano mínimo. Porém os estudos não mostram uma discrepância assim tão favorável. É notório, pela evidência objetivada e avaliada por autores especializados e independentes, que existe um balanço subjacente de prós e contras em torno desse rastreamento ${ }^{1,7,13,14}$. O papel de salvaguardar o equilíbrio dessa balança pertencerá, talvez, ao Médico de Família, cabendo então à mulher candidata colocar o último peso sobre um dos dois pratos: se essa mulher destaca que a sua evitação da morte por câncer de mama é uma prioridade, se tem elevado grau de receio de neoplasia mamária ou se a entrada no screening lhe traz tranquilidade, entáo provavelmente irá colocar o peso sobre o "prato dos prós"; se, pelo contrário, deseja sobretudo evitar a ansiedade oriunda de possíveis falso-positivos, se pretende contornar o risco de ter um tumor sobrediagnosticado ou se rejeita totalmente a exposição à radiaçáo, a exames histológicos de esclarecimento ou a intervenção cirúrgica que possa mutilar sua autoimagem, então provavelmente essa mulher terá tendência para pesar mais sobre o "prato dos contras", 122,14.

Esta decisão partilhada entre o médico e a mulher quanto ao início ou não do rastreamento constitui o verdadeiro paradigma da medicina centrada na pessoa, tão fiel aos valores de cada mulher única e táo facilitadora do exercício de algumas das competências nucleares do MFC: a abordagem holística, a prática de cuidados moldados à individualidade interpessoal e a prevenção quaternária ${ }^{17}$.

\section{Considerações finais}

A leitura detalhada da evidência científica implicará, talvez, na necessidade de acender a luz amarela no semáforo do rastreamento do câncer de mama. Não existe, no momento atual, um inequívoco saldo negativo no risco/benefício para acender a luz vermelha. Porém, poder-se-á manter a luz verde para o screening? Essa luz verde está claramente acesa no rastreamento em massa (mass screening) patrocinado pelos governos nacionais ou regionais ou nas recomendações provindas desses mesmos que sejam estímulo à prática de uma atitude clínica plenamente proativa (como o Plano de Fortalecimento da Rede de Prevenção, Diagnóstico e Tratamento do Câncer brasileiro ou o Plano Nacional de Prevenção e Controle das Doenças Oncológicas português $)^{9,10}$. Nesse último caso, uma decisão partilhada poderá sempre ter início numa oportunidade casual de explicação do rastreamento. A mulher poderá ter dúvidas e as suas questôes individuais relembram, por sua vez, as particularidades dessa pessoa, convidando à apresentação dos benefícios e riscos conhecidos. Porém, no caso do mass screening (que incluirá o convite não personalizado das mulheres em idades-alvo) poderá haver o borramento da individualidade de cada mulher e de um consenso que é estabelecido em consulta com seu médico.

Os defensores do rastreamento em massa alegam que um screening organizado e centralizado garante uma maior homogeneidade e qualidade dos métodos usados devido ao uso de recursos humanos altamente especializados (com redução de falso-positivos), algo que poderá minimizar um possível dano ${ }^{16}$. Contudo, paira a questáo de se a ultrapassagem da linha comunicacional que se estabelece entre a mulher e o seu médico cuidador náo será o abandono de uma medicina centrada na pessoa, tão importante numa área onde, mais do que nunca, existe a dúvida. 


\section{Referências}

1. Independent UK Panel on Breast Cancer Screening. The benefits and harms of breast cancer screening: an independent review. Lancet. 2012; 380(9855): 1778-86. http://dx.doi.org/10.1016/S0140-6736(12)61611-0

2. Bull M, Schröder FH. Screening for prostate cancer - the controversy continues, but can it be resolved?. Acta Oncol. 2011; 50(Suppl 1): 4-11. http:// dx.doi.org/10.3109/0284186X.2010.522197

3. Welch HG, Black WC. Overdiagnosis in cancer. J Natl Cancer Inst. 2010; 102: 605-13. http://dx.doi.org/10.1093/jnci/djq099

4. Gérvas J. Abuso de la prevención clínica. El cribaje del cáncer de mama como ejemplo. Rev Espaço Saúde. 2009; 11: 49-53

5. Carlsson S, Vickers AJ, Roobol M, Eastham J, Scardino P, Lilja H et al. Prostate Cancer Screening: Facts, Statistics and Interpretation in Response to the US Preventive Services Task Force Review. J Clin Oncol. 2012; 21(30): 2581-84. http://dx.doi.org/10.1200/JCO.2011.40.4327

6. Moyer VA, U.S. Preventive Services Task Force. Screening for prostate cancer: U.S. Preventive Services Task Force Recommendation statement. Ann Intern Med. 2008; 149: 185-91

7. Gotzsche PC, Hartling OJ, Nielsen M, Brodersen J. Screening for breast cancer with mamography. The Nordic Cochrane Centre; 2012. Disponível em: http://www.cochrane.dk/screening/mammography-leaflet.pdf

8. Cancer Research UK. Predicted improvements in breast cancer survival. Disponível em: http://www.cancerresearchuk.org/cancer-help/about-cancer/ cancer-questions/predicted-improvements-in-breast-cancer-survival

9. Brasil. Ministério da Saúde. Coordenação Nacional para as Doenças Oncológicas. Plano Nacional de Prevenção e Controlo das Doenças Oncológicas 2007-2010 Programa de desenvolvimento. Brasília: Ministério da Saúde, Alto Comissariado da Saúde; jul. 2009. Disponível em: http:// www.portaldasaude.pt/NR/rdonlyres/F2CE0CE2-9802-473D-9F45-F375687EB4F0/0/pnpcdoversaofinal8julho2009.pdf

10. Brasil. Ministério da Saúde. Fortalecimento da rede de prevenção, diagnóstico e tratamento do câncer. Brasília: Governo Federal; 2011. Disponível em: http://portal.saude.gov.br/portal/arquivos/pdf/2b_060511.pdf

11. Instituto Nacional de Câncer. Parâmetros técnicos para o rastreamento do câncer de mama Recomendações para gestores estaduais e municipais. Brasília: Ministério da Saúde; 2009. Disponível em: http://www.acervo.epsjv.fiocruz.br/beb/textocompleto/009471

12. National Center for Health Promotion and Disease Prevention. Discussing breast Cancer screening decisions with average risk women in their 40's. National Center for Health Promotion and Disease Prevention; 2011. Disponível em: http://www.prevention.va.gov/docs/VANCP_BreastCancerFact.pdf

13. Gotzsche PC, Nielsen M. Screening for breast cancer with mamography. Cochrane Database Syst Rev. 2011 Jan; (1):CD001877. http://dx.doi. org/10.1002/14651858.CD001877.pub4

14. Nelson HD, Tyne K, Naik A, Bougatsos C, Chan BK, Humphrey L. Screening for breast cancer:an update foto the US Preventive Services Task Force. Ann Intern Med. 2009; 151: 727-737

15. Thornton H, Pillariesetti RR. "Breast awareness" and "breast self-examination" are not the same. What do these terms mean? Why are they confused? Why can we do?. Eur J Cancer. 2008; 44(15): 2118-21. http://dx.doi.org/10.1016/j.ejca.2008.08.015

16. ARS Norte. Circular Normativa no 1 Prescrição de mamografia de rastreio em mulheres do grupo erário dos 45 a 69 anos, nas unidades de cuidados de saúde primários. ARS Norte 2011. Disponível em: http://portal.arsnorte.min-saude.pt/portal/page/portal/ARSNorte/Documentos/Circulares\%20 da\%20ARSNorte/Circulares\%20Normativas\%202011/Circular\%20Normativa\%201_2011.pdf

17. World Organization of Family Doctors - Wonca Europa. A definição europeia de Medicina Geral de Familiar. Wonca Europa; 2002. p. 6-14. 